Ann. Biol. anim. Bioch. Biophys., 1976, 16 (4), 623-629.

\title{
INFLUENCE DES CONDITIONS DE NUTRITION SUR LA QUALITÉ ET LA QUANTITÉ DE L'URINE DU RAT
}

\author{
J. CAMBAR \\ Laboratoire de Pharmacodynamie et Hydrologie, \\ Université Bordeaux II, \\ 91, rue Leyteire, \\ 33000 Bordeaux \\ RÉSUMÉ
}

Le présent travail se propose d'étudier l'influence des conditions de nutrition sur divers paramètres urinaires, tels le volume, la pureté, le taux en protéines et le poids de matières lyophilisées. L'étude des variations de ceux-ci est suivie pendant trois périodes successives de huit heures. L'urine collectée chez les animaux privés de nourriture, conditions auxquelles sont souvent soumis les animaux, présente de très importantes variations tant quantitatives que qualitatives.

Les protocoles expérimentaux suivis par les rares chercheurs nous ayant précédé dans l'étude de la protéinurie physiologique du Rat (SELLERS et coll., I950, I952 ; CoLLIOT, I972) nous ont semblé trop différentes des conditions dans lesquelles ce mammifère de laboratoire vit normalement. En effet, les animaux d'expérience sont privés de nourriture peu de temps avant le début du recueil des urines et pendant toute leur collecte. La justification du choix d'un tel protocole n'étant pas explicitée, nous avons essayé d'analyser l'influence des conditions de nutrition imposées aux rats pendant le recueil de l'urine sur certains paramètres urinaires importants, tels le volume, la pureté, le taux en protéines et le poids de lyophilisat, nouveau para mètre récemment introduit par nous-mêmes (CAMBAR, I974). 


\section{MATÉRIEI， ET MÉTHODE}

\section{I. - Appareillage utilisé pour la collecte des urines}

Les animaux d'expérience sont placés dans des cages à métabolisme en acier inoxydable (Matériel René Pajon, 45000 Orléans) munies d'un dispositif spécial classique, permettant de séparer les urines des fèces. Des précautions techniques sont indispensables pour limiter au maximum les risques de souillure de quelque nature qu'elle soit.

\section{2. - Conditions de nutrition}

Chaque expérience utilise un lot homogène de 5 rats blancs, lot d'animaux de même souche, même poids $(400 \mathrm{~g})$, même sexe (masculin). Chaque animal est isolé dans une cage à métabolisme et successivement soumis à trois conditions de nutrition différentes :

- Conditions $A$ : nourriture et boisson ad libitum pendant toute la durée de la collecte de l'urine, ainsi que pendant les 24 heures qui précèdent. Ce sont donc les conditions normales de nutrition des animaux.

- Conditions $B$ : privation de nourriture pendant la durée de la collecte des urines. La nourriture et la boisson sont fournies ad libitum pendant les 24 heures qui précèdent la collecte.

- Conditions $C$ (seulement pour l'étude du volume urinaire) : privation de nourriture pendant la durée de recueil des urines $(24$ heures), ainsi que pendant les 24 heures qui précèdent. L'eau de boisson est donnée ad libitum.

\section{3. - Purification et traitement des urines}

Dès leur recueil, les urines sont purifiées par filtration sous-pression sur des pré-filtres Millipore (diamètre des pores : 5 microns).

Le volume urinaire est directement mesuré.

Les pré-filtres, préalablement tarés, ont retenu les produits insolubles; ils sont desséchés à l'étuve, puis pesés. Le poids sec des produits insolubles est ainsi mesuré.

Les lyophilisats urinaires sont obtenus par la méthode déjà décrite (CAMBAR, 1974). A partir des lyophilisats d'urines récoltés pendant 8 ou 24 heures, on détermine la quantité de lyophilisat $L$ éliminée et le taux $T$, quantité $L$ éliminée par millilitre d'urine, qui est donc la teneur en matières sèches déterminée par lyophilisation des volumes d'urine.

Enfin, les protéines urinaires totales sont dosées directement sur les lyophilisats urinaires par la méthode classique de Gornall (I949), mođifiée par Cambar (1974).

\section{RÉSULTATS}

\section{I. - Etude des variations du volume urinaire}

Un jeûne prolongé (conditions C) exerce une influence sur le volume urinaire (tabl. I) ; ce volume atteint, à peine, la moitié de celui mesuré dans les conditions A ou B. Au contraire, aucune différence significative n'a été mise en évidence entre les volumes urinaires obtenus dans les conditions A ou B. 


\section{TABLEAU I}

Exemple de variations du volume urinaire, selon différentes conditions de nutrition (en $\mathrm{ml}$ par 24 heures)

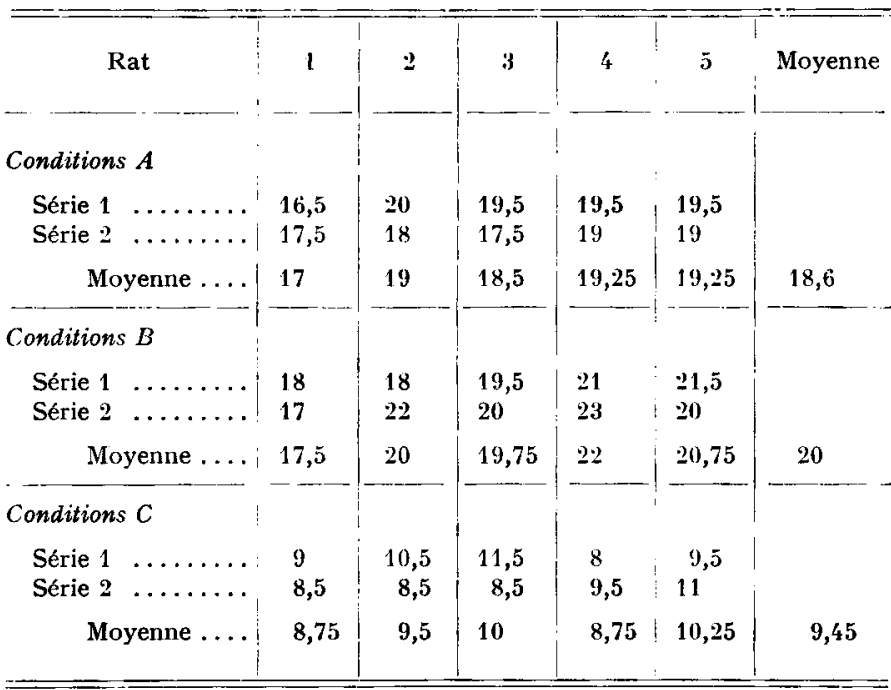

2. -- Étude des variations du poids des produits insolubles

Si les rats absorbent de la nourriture pendant le recueil des urines (conditions A), celles-ci sont beaucoup plus riches en produits insolubles (tabl. 2 ).

\section{TABIEAU 2}

Exemple de variations du poids des substances insolubles contenues dans l'urine de rats soumis à deux conditions de nutrition différentes

(en $\mathrm{mg}$ par 24 heures)

\begin{tabular}{|c|c|c|c|c|c|c|}
\hline Rat & I & 2 & 3 & 4 & 5 & Moyenne \\
\hline \multicolumn{7}{|l|}{ Conditions $A$} \\
\hline Série 1 . & 59 & 64 & 82 & 68 & 86 & \\
\hline Série 2. & 85 & 80 & 108 & 99 & 76 & \\
\hline Moyenne .... & 72 & 72 & 95 & 83,5 & 81 & 80,7 \\
\hline \multicolumn{7}{|l|}{ Conditions $B$} \\
\hline Série $1 \ldots \ldots \ldots$ & 49 & 41 & 42 & 34 & 78 & \\
\hline Série $2 \ldots \ldots \ldots$ & 38 & 43 & 53 & 38 & 50 & \\
\hline Moyenne... & 43,5 & 4: & 47,5 & 36 & 64 & 46,6 \\
\hline
\end{tabular}


Cette constatation était prévisible. Néanmoins, si la diminution de la quantité des produits insolubles est considérable, il est surprenant de constater la persistance d'une quantité importante de substances insolubles (de l'ordre de $40 \mathrm{mg} / 24$ heures) dans les urines de rats non nourris (conditions $B$ ). Cette remarque suggère la présence de produits insolubles d'origine urinaire, ajoutés aux impuretés d'origine alimentaire.

L'observation macroscopique des dépôts urinaires recueillis dans les conditions A fournit une première indication concernant cette double origine. Il existe, en effet, une différence de couleur et de vitesse de sédimentation entre les grains bruns déposés au fond du tube et le précipité blanchâtre floconneux surnageant. Puisque les grains bruns disparaissent dans les conditions $B$ à la différence du précipité floconneux qui persiste, ils nous paraissent être d'origine alimentaire. L'emploi de thymol, dans tous les cas, nous interdit d'assimiler le précipité blanchâtre à une prolifération bactérienne. Les résultats négatifs de l'étude électrophorétique et les traces de protéines dosées montrent à l'évidence l'origine non protéique de ce précipité. Eñin, la dissolution d'une partie du précipité dans l'acide acétique permet de supposer la présence de quantités importantes de phosphates alcalins, hypothèse confirmée par la mesure du $\mathrm{pH}$ urinaire alcalin et par microcristallographie.

\section{3. - Les variations du poids des lyophilisats}

Le poids des lyophilisats a été mesuré sur les urines de 24 heures de Rat soumis aux conditions A et B (cf. tabl. 3). Ce poids $\mathrm{L}$ est considérablement accru lorsque les animaux sont nourris (conditions A).

\section{TABLEAU 3}

Variations du poids des lyophilisats urinaires en fonction des conditions de nutrition

(en mg par 24 heures)

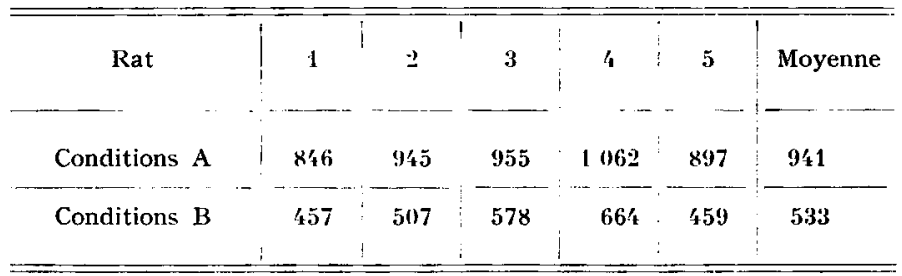

L'étude comparative des variations nycthémérales (pendant 3 périodes consécutives de 8 heures) de la quantité de lyophilisat par millilitre d'urine (appelé taux T, en $\mathrm{mg} / \mathrm{ml}$ ) fait apparaître les mêmes différences que celles enregistrées avec $L_{\text {, }}$, suivant les conditions A ou B (fig. I).

La sensible différence existant entre les poids $\mathrm{L}$ recueillis dans la période $8 \mathrm{~h}$ I6 $\mathrm{h}$ dans les conditions A et B semble surprenante si l'on considère la faible activité nutritionnelle diurne admise des animaux mais montre à l'évidence les graves répercussions de l'absence de nourriture même en période de jour.

De plus, dans les conditions $B$, on note un appauvrissement régulier de l'urine en corps dissous, appauvrissement s'expliquant par le manque de nourriture, alors 
que chez les animaux normalement nourris, nous observons un accroissement du taux T pendant la période de recueil nocturne, accroissement dû à la grande activité nutritionnelle des rats pendant la période d'obscurité.

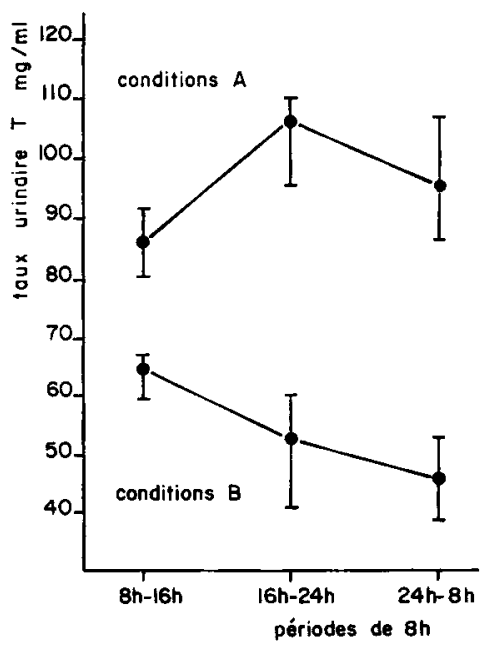

Fig. I. - Variations du taux de lyophilisat dans l'urine de 5 rats, soumis aux conditions de nutrition $A$ et $B$, pendant 3 périodes successives de 8 heures

\section{4. - Les variations de la protéinurie}

On observe une très nette augmentation de la protéinurie dans les conditions de nutrition A (tab1. 4), la quantité de protéines totales émises en 24 heures étant presque deux fois supérieure à celle émise dans les conditions de nutrition $B$.

\section{TABLEAU 4}

Protéinurie par 24 heures (en $\mathrm{mg}$ ) chez 5 rats mesurée sur les lyophilisats d'urine

\begin{tabular}{c|c|c|c|c|c|c}
\hline \hline Rat & 1 & 2 & 3 & 1 & 5 & Moyenne \\
\hline & & & & & & \\
\hline \\
Conditions B & 8,9 & 10,0 & 11,9 & 8,1 & 10,4 & 9,8 \\
\hline Conditions A & 17,9 & 19,6 & 20,9 & 16,1 & 20,1 & 18,9 \\
\hline
\end{tabular}

\section{DISCUSSION}

La très nette diminution du taux des lyophilisats et des protéines totales de l'urine des rats soumis au jeûne partiel (conditions $B$ ) montre d'une manière indiscutable que l'étude détaillée des constituants urinaires doit tenir compte des conditions 
de nutrition des animaux d'expérience et que, plus précisément, ces derniers doivent être normalement nourris. Il semble donc, que seules des raisons de simplicité et de rapidité de purification des urines aient conduit certains auteurs à préconiser un mode expérimental différent.

C'ertes, malgré toutes les précautions prises, on peut supposer que l'urine d'animaux normalement nourris risque d'être souillée et, de ce fait, contenir une quantité d'impuretés non négligeable. Or, les urines doivent être très limpides et peu souillées en vue de leur lyophilisation et de leur traitement ultérieur. Toutefois, à notre avis, les risques de pollution de l'urine par les fèces sont pratiquement écartés grâce à l'utilisation des cages à métabolisme particulières, déjà décrites. Une autre objection doit être levée : celle de la possibilité d'une pollution protéique de l'urine d'origine alimentaire, fondée sur la présence de certains fragments pulvérulents de nourriture dans les tubes collecteurs. Pour cela, nous avons effectué de nombreuses expériences consistant à dissoudre des quantités variables de nourriture pulvérisée très finement, dans des volumes aqueux comparables à ceux de l'urine émise.

Nous avons ainsi recherché le taux de pollution et montré qu'il était de l'ordre de 7 à $8 \mathrm{p}$. IOO, parmi lesquels I,25 p. roo seulement était d'un poids moléculaire supérieur à la limite supérieure des protéines retenues sur les ultra-filtres moléculaires utilisés pour purifier les urines. C'est pourquoi, nous pouvons conclure que, dans les conditions d'étude que nous préconisons, la pollution protéique par la nourriture des animaux est très faible, voire négligeable.

\section{CONCLUSION}

Le présent travail met donc en évidence que toute étude de l'urine de Rat et plus particulièrement de la protéinurie physiologique doit se faire chez des animaux en expérience, soumis aux conditions normales de nutrition, sous peine d'introduire d'importants risques d'erreurs.

Reçu pour publication en octobre 1975.

\section{SUMMARY}

\section{EFFECTS OF NUTRITION CONDITIONS ON QUALITY AND QUANTITY OF RAT URINE}

This paper studies the effect of nutrition conditions on different urinary factors in rat such as volume, purity, protein pattern and lyophilized matter. The variation of these factors is observed during three eight-hour periods. Urine collected from animals deprived of food (often the case) presents large quantitative and qualitative variations. 


\section{RÉFÉRENCES BIBLIOGRAPHIQUES}

САMBAR J., 1974. Contribution à l'étude de la filtration glomérulaire des macromolécules protéiques chez le Rat blanc. Thèse de Doctorat en Sciences Biologiques, Bordeaux $n^{\circ}$ I 139 .

Corliot J. E., 1972. Étude electrophorétique et bibliographique de la protéinurie physiologique chez le Rat. Thèse de Docteur Vétérinaire, Lyon $n^{\circ} 79$.

Gornall A. G., Badawill C. J., David M. M., I949. Determination of serum proteins by means of the biuret reaction. J. Biol., 177, 75I.

Sellers A. L., Goodman H. C., Marmorston J., Smith M., 1950. Sex difference in proteinuria in the Rat. Amer. J. Phys., 163, 662-667.

Sellers A. L., Sidney Roberts P. D., Rask I., Smith S., Goodman H. C., 1952. An electrophoretic study of urinary protein in the Rat. J. Exp. Med., 95, 465-472. 